\title{
The mental health impact of bed bug infestations: a scoping review
}

\author{
Rachelle Ashcroft • Yukari Seko • Lai Fong Chan • Jessica Dere • \\ Jaemin Kim • Kwame McKenzie
}

Received: 16 February 2015 / Revised: 30 June 2015 / Accepted: 3 July 2015 / Published online: 23 August 2015

(C) Swiss School of Public Health 2015

\begin{abstract}
Objectives We conducted a scoping review to identify and summarize the current state of knowledge regarding the mental health effects associated with bed bugs.

Methods We employed a five-stage scoping review framework, to systematically identify and review eligible articles. Eligibility criteria included a focus on bed bug infestations and reference to mental health impacts. Descriptive information was then extracted from each article, including the specific mental health effects cited. Results An initial search yielded 920 unique articles on the topic of bed bugs. Of these, 261 underwent abstract review, and 167 underwent full-text review. Full-text review and subsequent review of reference lists yielded a final sample of 51 articles. Numerous mental health effects were linked to bed bug infestations, including severe psy-
\end{abstract}

This review is part of the special issue "Driving the Best Science to Meet Global Health Challenges" edited on the occasion of the 9th European Congress on Tropical Medicine and International Health 2015 .

R. Ashcroft and Y. Seko contributed equally to this work.

R. Ashcroft ( $\square)$

Renison University College, University of Waterloo,

Waterloo, Canada

e-mail: rachelle.ashcroft@uwaterloo.ca

Y. Seko

University of Guelph, Guelph, Canada

L. F. Chan

National University of Malaysia Medical Centre (UKMMC),

Kuala Lumpur, Malaysia

J. Dere

University of Toronto Scarborough, Toronto, Canada chiatric symptoms. However, the majority $(n=31)$ of the articles were commentary papers; only five original research articles were identified.

Conclusions Although significant mental health effects are often linked to bed bugs, such discussions remain largely anecdotal. Despite recognition that the impact of bed bugs constitutes an important public health concern, little empirical evidence currently exists on this topic.

Keywords Mental health - Bed bugs - Bedbugs · Infestation

\section{Introduction}

The common bed bug, cimex lectularius, is a human parasite that has been in existence for thousands of years (Goddard and de Shazo 2009). Bed bugs are wingless, oval-shaped, flat, reddish-brown insects that grow to an adult length of approximately $5 \mathrm{~mm}$ (Goddard and de Shazo 2009; Pritchard and Hwang 2009). They resemble small cockroaches or unfed ticks (Goddard and de Shazo 2009). During the day, bed bugs hide in cracks found in

\author{
J. Kim \\ Cancer Care Ontario, Toronto, Canada \\ K. McKenzie \\ Centre of Addictions and Mental Health, Toronto, Canada \\ K. McKenzie \\ University of Toronto, Toronto, Canada \\ K. McKenzie \\ Wellesley Institute, Toronto, Canada
}


beds, furniture, flooring, and walls (Pritchard and Hwang 2009). Bed bugs appear at night to feed (Pritchard and Hwang 2009) and feed entirely on blood (Eddy and Jones 2011). Typically, bed bugs feed when the host is asleep by biting a host's exposed areas such as arms, legs, neck, and face (Eddy and Jones 2011). Feeding sessions are approximately 10-20 min in duration (Davies et al. 2012). Afterwards, bed bugs may increase by $30-50 \%$ in length and 150-200\% in weight (Goddard and de Shazo 2009). "Indications of an infestation include unexplained bite reactions, dark spotting on the bed from faecal deposition, and presence of the insects themselves" (Doggett and Russell 2009, p. 881).

Bed bugs constitute an emerging public health problem worldwide (Goddard and de Shazo 2009; Huntington 2012), with increasing reports of bed bug infestations across diverse settings, including homes, apartments, healthcare facilities, and hotels (Goddard and de Shazo 2009). Since the mid-1990s, there has been a global resurgence of bed bugs (Doggett and Russell 2009), with reports confirming a rising incidence of infestations (Pritchard and Hwang 2009). For example, infestation rates in Australia rose by $4500 \%$ between 2000 and 2006 (Doggett and Russell 2009, p. 880). Metropolitan centers in North America have witnessed similarly dramatic increases in infestation rates. Toronto Public Health has no recorded numbers of bed bug infestations occurring before 2003, and in 2003 only 46 accounts of bed bugs were reported (Woodgreen Community Services 2009). By 2008, however, Toronto Public Health received 1500 reports of bed bug infestations between March and October alone (Woodgreen Community Services 2009). New York City has even higher reported levels of bed bug infestations. In 2003, for example, there were 537 reports made to the city's telephone complaint line. In 2004, there were 1839 complaints of bed bug infestations, which rose to 10,985 reports in 2009 (Ralph et al. 2013). The major resurgence of bed bugs is "leading to clinical and control problems" (Doggett et al. 2012, p. 164), to the extent that bed bug infestations have been described as a "global epidemic" (Aultman 2012).

With bed bugs becoming increasingly problematic, it appears likely that there are physical and mental health consequences for those whose lives are impacted by an infestation. Insects can evoke a sense of distress, fear, anxiety, increased heart rate, and impairment in functioning for some individuals (Stoyanova and Hope 2012). For example, head lice do not transmit disease yet can be a cause of considerable mental and social distress (Chunge et al. 1991). Head lice infestations in children can result in feelings of low self-esteem, stigma, and high levels of anxiety for both children and their parents (Frydenberg and Starr 2003; Villegas 2012). Furthermore, flea infestation and bites have been found to cause "significant psychological distress" (Naumann et al. 2011, p. 226) and to negatively impact mood and morale (Naumann et al. 2011). Bed bug infestations may similarly lead to mental health consequences, such as nervousness, anxiety, and insomnia (Thomas et al. 2013). However, despite being an emerging public health problem, little is actually known about the impact of bed bugs on mental health. This scoping review, therefore, aims to advance our knowledge about an increasingly important topic for mental health research. We present the current state of knowledge regarding the mental health effects associated with bed bugs, and identify areas where future research is needed.

\section{Methods}

Research question and scope

We employed a five-stage scoping review framework proposed by Arksey and O'Malley (2005), which consists of the following stages: (1) identifying the research question; (2) identifying relevant studies; (3) study selection; (4) charting the data; and (5) collating, summarizing and reporting the results. This method is designed to systematically map the subject field and is considered necessary when literature on a topic is being compiled for the first time, when limited literature exists for a given topic, and/or when the topic under investigation is complex or non-homogeneous (Grimshaw 2010; Shankardass et al. 2011). This framework was considered optimal for the current study, due to the infancy of the subject matter and scarcity of evidence-based studies. At the first stage, the overarching question guiding this study was: "what is the current state of knowledge regarding the mental health effects of bed bug infestations?"

\section{Publication selection and data extraction}

At stage two, we sought to identify all available literature on bed bug infestations published in English prior to May 2013. The databases and keywords were determined in consultation with a senior librarian at the Centre for Addictions and Mental Health (CAMH) in Toronto, Canada. We conducted a comprehensive search of MEDLINE, EMBASE, CINHAL, and PsycINFO using the search terms 'bed bug*', 'bedbug*', and 'cimex lectularius*' from the inception of each database until the fourth week of May 2013. Duplicates of articles were removed.

At stage three, five authors (RA, LFC, JD, JK, and YS) independently conducted title scans and abstract reviews to assess eligibility against the following inclusion criteria: (1) the word bed bugs or bedbugs or cimex lectularius are used in the article; (2) the article focuses primarily on bed 
bug infestations; (3) the authors are academic researchers and/or professional associations; (4) the article refers to mental health impacts on human beings; and (5) the article is written in English. Gray literature (e.g., dissertations and theses, government documents, conference proceedings and posters) and popular media coverage were excluded. Following the abstract review, we then reviewed the full texts of those articles that passed the initial screening, using the same inclusion criteria. Following a recommendation by Levac et al. (2010), we employed a team approach in the process of literature screening. We discussed data characterization and extraction methods as a team at the beginning, during the middle, and at the end of the review process until a consensus was reached.

At stage four, we independently extracted the following data from all studies included in the scoping review: authorship, year of publication, geographical origin of study or article, type of article (e.g., original study, commentary paper), and description of mental health effects. As the focus of our scoping review is the current state of knowledge regarding mental health effects of bed bugs, we sought to summarize the types of effects cited in the articles in a structured way. A diathesis-stress model guided our categorization of the effects cited in the articles (Goforth et al. 2011). Symptoms of psychological distress may be an acute reaction to a stressful situation. When an individual is able to cope, those transient symptoms resolve and normal functioning is regained. In vulnerable populations-for example, individuals who are genetically predisposed to mental illness, or who have additional psychosocial stressors-these symptoms may progress to more serious psychiatric manifestations (e.g., aggression, psychosis, suicidal behavior). The stressor (e.g., bed bug infestation) may also act as a precipitating or perpetuating factor in the onset or worsening of diagnosable psychiatric disorders (Goforth et al. 2011). Therefore, we categorized the mental health effects of bed bug infestation into three categories, (1) general psychological symptoms or distress, (2) serious psychiatric manifestations (psychotic symptoms-commonly associated with schizophrenia and/or psychotic disorders-and manifestations of serious mood disorders); and (3) specific reference to psychiatric disorders. Our approach to categorization is consistent with Health Canada (2002) and Davis (2013), who define serious and persistent mental disorders as referring to schizophrenia and serious mood disorders such as bipolar disorder.

\section{Results}

Following these four steps generated a breadth of findings. The initial database search yielded 920 unique articles and all references were imported into the bibliographic management program EndNote (version X4) for relevance screening. Of the articles collected, 261 abstracts were selected for further abstract review and 167 articles were selected for full-text review. After the full-text review, 50 articles met inclusion criteria. Following this process, five authors (RA, LFC, JD, JK, and YS) hand searched the reference lists of identified articles for any additional relevant articles and conducted a Google Scholar search to ensure an exhaustive search. One article was added in this step. A total of 51 articles were, therefore, included in the final stage of the scoping review (see Fig. 1).

\section{Characteristics of included articles}

Table 1 provides descriptive information for the 51 publications identified through our systematic search. During data characterization, we identified five original research papers (Gbakima et al. 2002; Goddard and de Shazo 2012; Goodall and Reed 2013; Potter et al.2010; Susser et al. 2012), six case reports (Burrows et al.2013; Cimolai and Cimolai 2012; Comack and Lyons 2011; Pritchard and Hwang 2009; Rieder et al. 2012; Ter Poorten and Prose 2005), seven literature reviews (Delaunay 2012; MunozPrice et al. 2012; Ratnapradipa et al. 2011; Shum et al. 2012; Singh and Mann 2013; Thomas et al. 2013; Williams and Willis 2012), two technical guidelines for health professionals (Armed Forces Pest Management Board 2010; Sciscione 2012), and the remaining 31 articles were commentary papers. Although the literature reviews contained content related to mental health impacts, the literature reviews themselves did not have an exclusive focus on the mental health impacts related to bed bug infestations. For example, Delaunay (2012) explored the topic of bed bugs and travel whereas MunozPrice et al. (2012) explored the topic of bed bugs in healthcare settings.

All publications were published between 2002 and 2013, with $74.5 \%$ (38 out of 51) published after 2010. The majority of publications were written by researchers in North America $(n=39)$, with the remaining articles written by authors in the U.K. $(n=3)$, France $(n=3)$, Australia $(n=2)$, Sierra Leon $(n=1)$, Brazil $(n=1)$, Israel $(n=1)$, and India $(n=1)$. The authors of the included articles have diverse disciplinary backgrounds, including public health, nursing, family medicine, dermatology, pharmacy, and medical entomology. Notably, only one article was written by psychiatrists (Rieder et al. 2012). In 47 papers $(92.1 \%)$, the primary focus was on public health impacts of bed bugs, with specific mentions of physical health consequences such as cutaneous reactions (e.g., erythematous papules and lesions, pruritic wheals, urticaria, bullous rashes), systemic reactions (e.g., asthma, 
Fig. 1 Scoping review methodology used to identify 51 articles in study sample

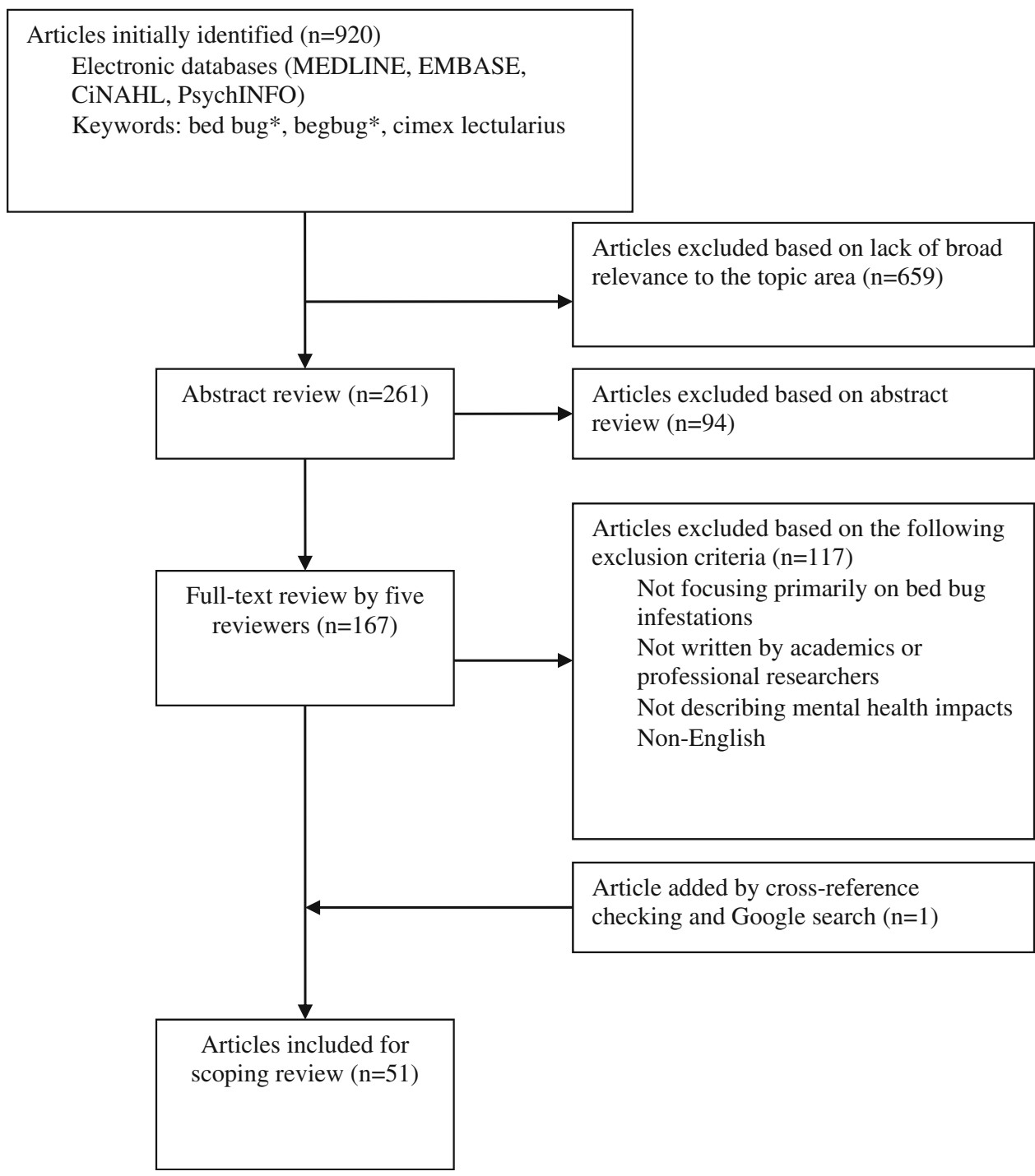

anaphylaxis) and in severe cases, anemia and skin leathering. Compared to the discussion of physical health impacts, references to psychological consequences of bed bug infestations were largely anecdotal in these articles.

Mental health impacts

All 51 articles mentioned some degree of general psychological distress. Diagnosable psychiatric disorders were described in $15(29.4 \%)$ articles and 7 (13.7\%) articles described serious psychiatric manifestations (see Figs. 2, 3 ). Five articles included in the review (Burrows et al. 2013; Goddard and de Shazo 2012; Rieder et al. 2012; Susser et al. 2012; Zipple et al. 2012) focused exclusively on mental health effects of bed bug infestations. Among these five articles, two included studies that utilized validated criteria from the fourth edition of the diagnostic and statistical manual of mental disorders (DSM-IV) to measure post-traumatic stress disorder (PTSD) symptoms (Goddard and de Shazo 2012) and depression and anxiety symptoms (Susser et al. 2012). Two articles were case reports (Burrows et al. 2013; Rieder et al. 2012). Burrows et al. (2013) present the case of a woman who jumped to her death where repeated bed bug infestations were considered to be the likely trigger for the psychological state that led to suicide. Rieder et al. (2012) present seven clinical cases of patients presenting with psychiatric consequences related to actual infestation and perceived or feared infestations.

Exposure to bed bug-infested environments has been associated with a variety of psychological consequences. Among the 51 publications, 28 (54.9\%) referred to psychological distress and stress caused by actual bed bug bites, as well as by nightmares and flashbacks following an infestation. Noting difficulty of eradicating bed bugs, 26 articles $(50.9 \%)$ asserted that victims of frequent bed bug 


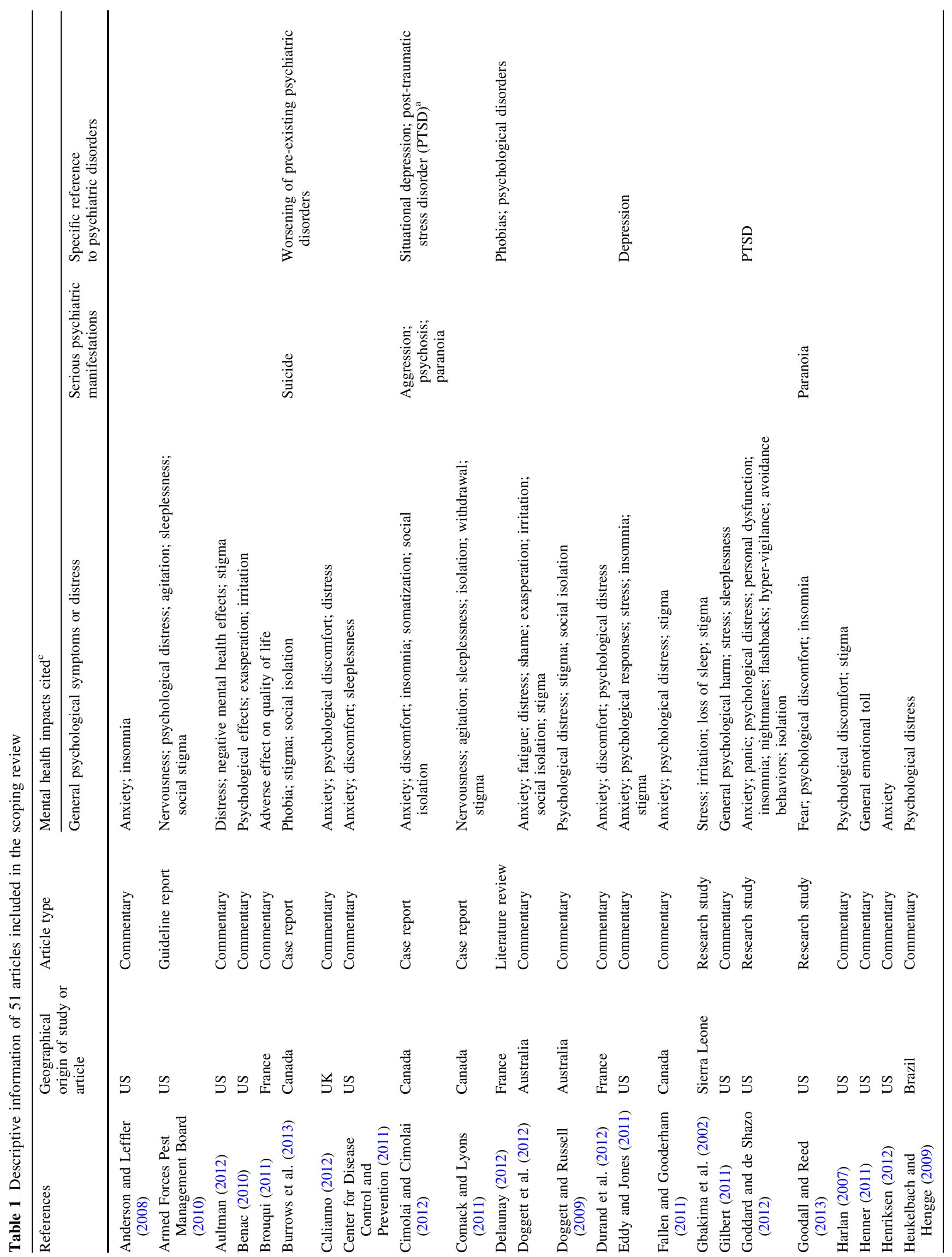




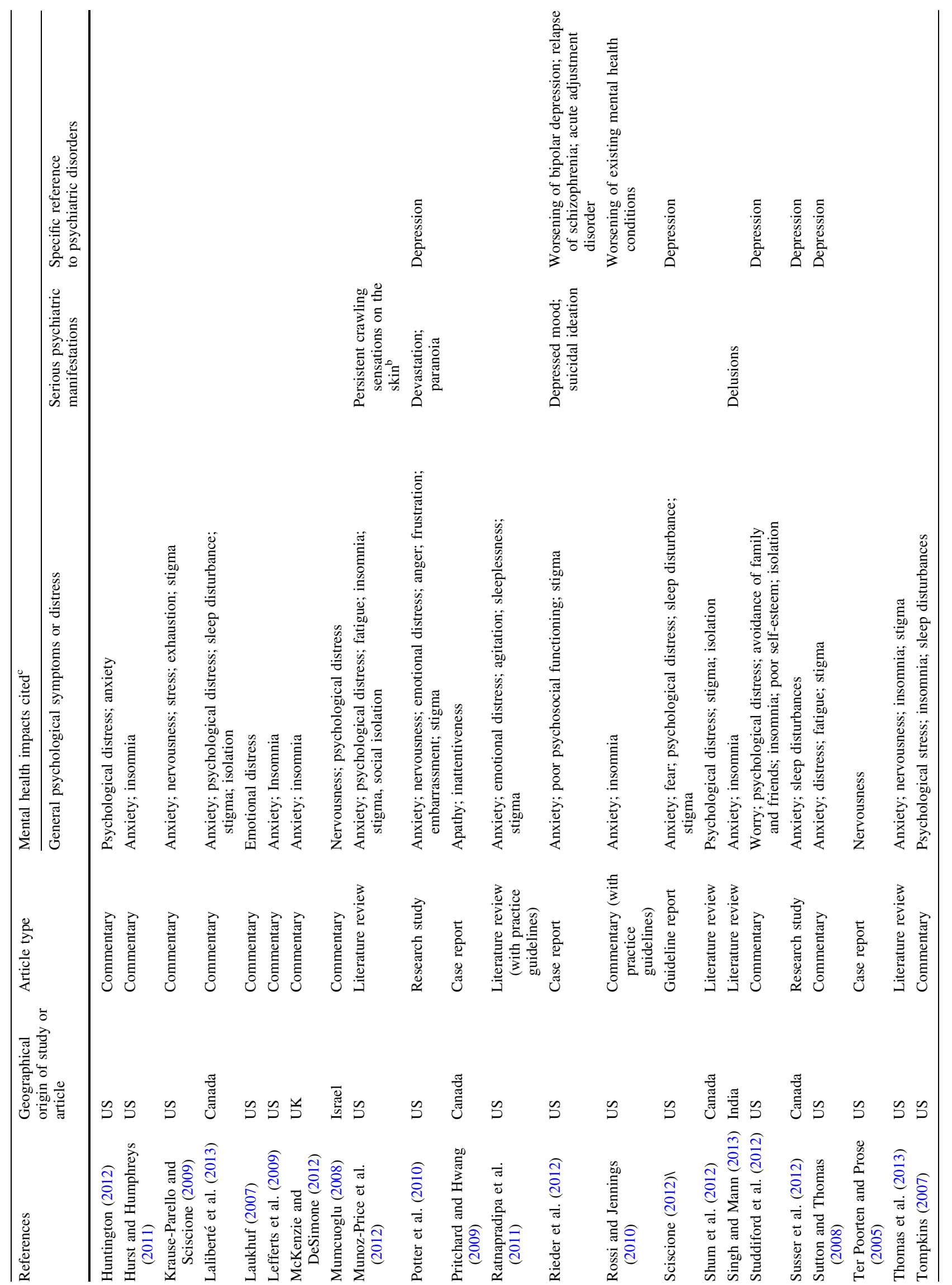



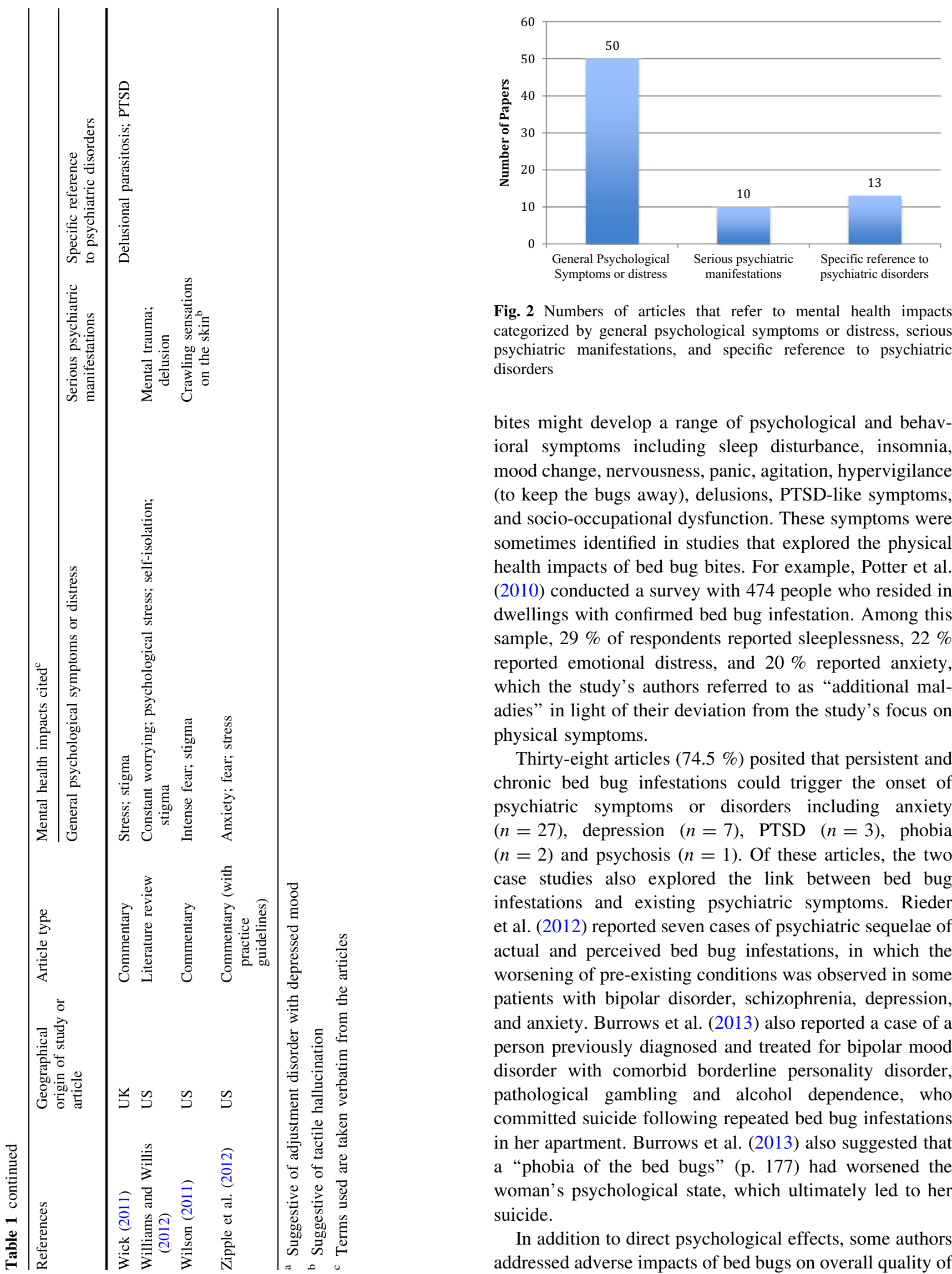

Fig. 2 Numbers of articles that refer to mental health impacts categorized by general psychological symptoms or distress, serious psychiatric manifestations, and specific reference to psychiatric disorders

bites might develop a range of psychological and behavioral symptoms including sleep disturbance, insomnia, mood change, nervousness, panic, agitation, hypervigilance (to keep the bugs away), delusions, PTSD-like symptoms, and socio-occupational dysfunction. These symptoms were sometimes identified in studies that explored the physical health impacts of bed bug bites. For example, Potter et al. (2010) conducted a survey with 474 people who resided in dwellings with confirmed bed bug infestation. Among this sample, $29 \%$ of respondents reported sleeplessness, $22 \%$ reported emotional distress, and $20 \%$ reported anxiety, which the study's authors referred to as "additional maladies" in light of their deviation from the study's focus on physical symptoms.

Thirty-eight articles $(74.5 \%)$ posited that persistent and chronic bed bug infestations could trigger the onset of psychiatric symptoms or disorders including anxiety $(n=27)$, depression $(n=7)$, PTSD $(n=3)$, phobia $(n=2)$ and psychosis $(n=1)$. Of these articles, the two case studies also explored the link between bed bug infestations and existing psychiatric symptoms. Rieder et al. (2012) reported seven cases of psychiatric sequelae of actual and perceived bed bug infestations, in which the worsening of pre-existing conditions was observed in some patients with bipolar disorder, schizophrenia, depression, and anxiety. Burrows et al. (2013) also reported a case of a person previously diagnosed and treated for bipolar mood disorder with comorbid borderline personality disorder, pathological gambling and alcohol dependence, who committed suicide following repeated bed bug infestations in her apartment. Burrows et al. (2013) also suggested that a "phobia of the bed bugs" (p. 177) had worsened the woman's psychological state, which ultimately led to her suicide.

In addition to direct psychological effects, some authors addressed adverse impacts of bed bugs on overall quality of 


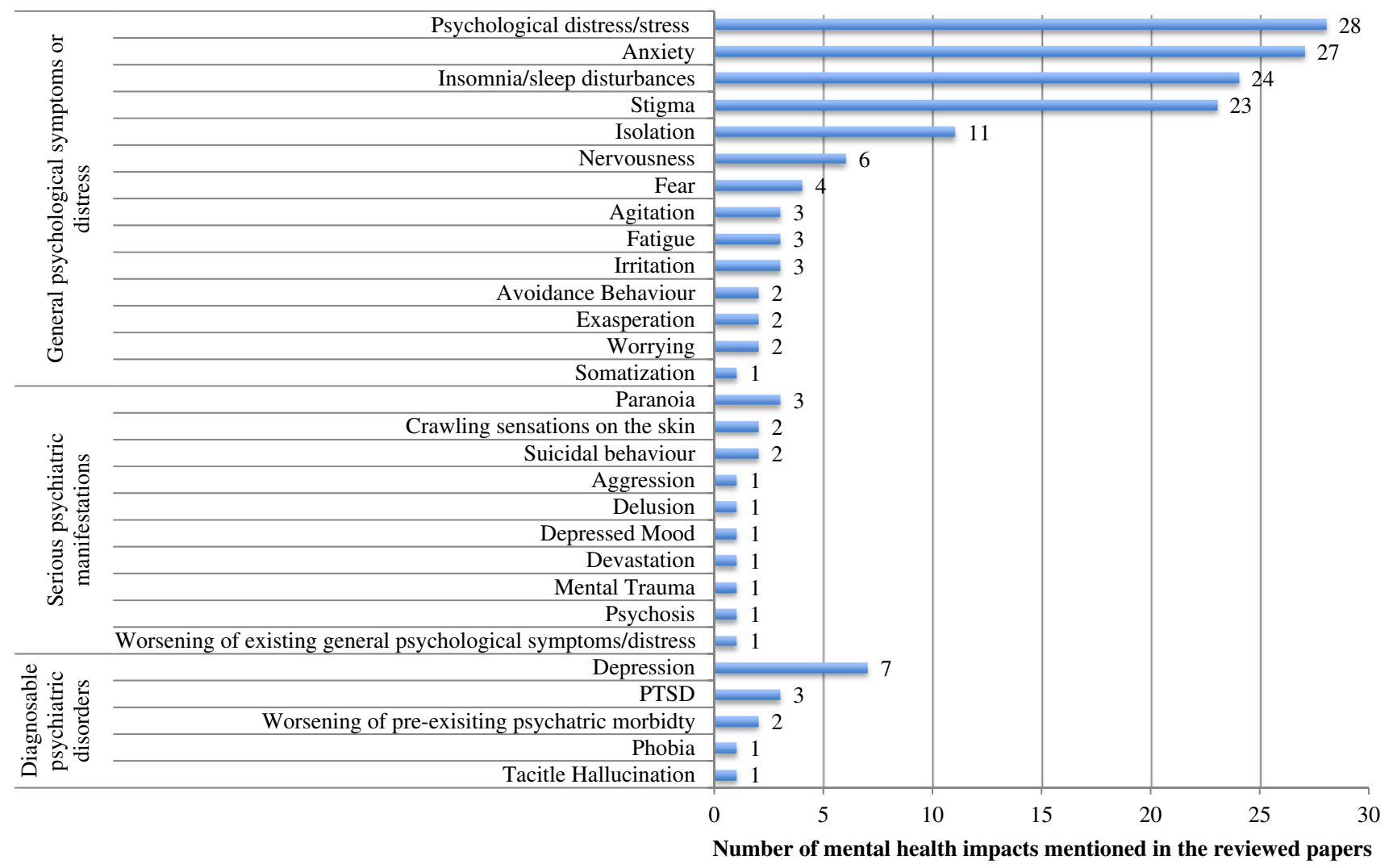

Fig. 3 Details of general psychological symptoms or distress, serious psychiatric manifestations, and specific reference to psychiatric disorders

life. Twenty-three papers $(45.1 \%)$ mentioned the notion that social stigma associated with bed bugs (e.g., misconceptions that infestations are related to poor hygiene) could cause shame and prevent victims from seeking help. Eleven articles $(21.6 \%)$ pointed out that social isolation caused by the stigma and fear of contamination could result in decreased self-esteem and further withdrawal from social networks. In 21 articles (41.2 \%), relevant economic consequences were discussed, including the financial burden incurred from extermination attempts, as well as secondary costs for the cleaning and replacement of clothing and furniture.

Authors commonly argued that eradication of bed bugs often requires a prolonged and costly approach and thus could cause additional psychological and economic anguish to victims. In this regard, some authors asserted that detrimental impacts of bed bugs could be more profound for vulnerable populations at greater risk of poorer health outcomes related to social determinants of health, such as people experiencing homelessness, the elderly (Eddy and Jones 2011; Laliberté et al. 2013; Aultman 2012), individuals with substance abuse (Pritchard and Hwang 2009), residents in refugee camps (Gbakima et al. 2002), and people with pre-existing mental illness (Rieder et al. 2012).

\section{Discussion}

To the best of our knowledge, the current scoping review represents the most comprehensive overview published to date of the psychological effects of bed bug infestations. Of the 51 articles in our review sample, only five are original research studies (Gbakima et al. 2002; Goddard and de Shazo 2012; Goodall and Reed 2013; Potter et al. 2010; Susser et al. 2012). Each of these five studies suggests that individuals residing in environments infested by bed bugs are at risk of suffering from a broad range of mental health effects, including insomnia, irritation, emotional distress, psychological distress and discomfort, fear, nightmares, flashbacks, hypervigilance, anxiety, panic, avoidance behaviors, PTSD, paranoia, and depression.

Strikingly, the majority of the articles examined in this scoping review are largely anecdotal and descriptive in nature. Although the articles refer to a range of psychological and psychiatric symptoms that may be caused by bed bugs, the authors rarely support their claims with scientific evidence. We are only beginning to understand the impact of bed bugs on mental health, despite the fact that bed bugs constitute a serious public health problem in Canada, the United States, and elsewhere in the world (Bernardeschi et al. 2013; Goddard and de Shazo 2009; 
Huntington 2012; Ralph et al. 2013). For instance, Huntington (2012) provides guidance for physicians to treat psychiatric symptoms related to bed bug infestations using cognitive behavioral therapy and pharmacotherapy, despite the lack of relevant evidence to support many of these claims. If such claims are accurate, then more evidence-based clinical guidance is required to address the mental health impacts of bed bug infestations (Doggett and Russell 2009; Pritchard and Hwang 2009; Goddard and de Shazo 2009).

One of these impacts includes the onset and/or worsening of psychiatric symptoms (Rieder et al. 2012; Burrows et al. 2013). General psychological symptoms or distress are identified the most frequently in our sample, followed by specific reference to psychiatric disorders, and serious psychiatric manifestations. Perhaps most concerning is that bed bugs may even increase the risk of suicide (Burrows et al. 2013). Pritchard and Hwang (2009) also suggest that cognitive impairments and serious or persistent mental illness may exacerbate the severity of a bed bug infestation because the person may not have the capacity to address the conditions in the home.

A number of the articles we reviewed suggest that vulnerable populations may be at greater risk for the detrimental mental health effects of bed bug infestations (Eddy and Jones 2011; Laliberté et al. 2013; Aultman 2012; Pritchard and Hwang 2009; Gbakima et al. 2002; Rieder et al. 2012). One of the reasons for this may be that vulnerable populations (such as people with unstable housing, racialized populations, and individuals with existing mental health conditions) are more likely to experience bed bugs infestations as an environmental hazard (Masuda et al. 2008). The correlation between harmful environmental factors and increased rates of related burden of disease is well known (Prüss-Üstün and Corvalán 2006). The World Health Organization (WHO) recognizes this correlation when it advocates improvements to housing and living conditions to enhance physical and mental health, and to decrease the environmental burden of disease (Prüss-Üstün and Corvalán 2006). Such a correlation (Baum 2003) makes it far more likely that individuals residing in bed bug-infested environments face adverse mental health impacts as a result. Thus, if vulnerable populations are experiencing the greatest mental health impacts of living within bed bug infestations, bed bugs may perpetuate health inequities. Providing adequate social support and mental health services to vulnerable populations to prevent social stigma and isolation should be as important a priority as eradicating the bed bugs themselves.

Another salient finding of our review was the interdisciplinary nature of interest in the mental health impacts of bed bugs because the articles we reviewed represented a wide range of disciplines, including public health, nursing, family medicine, dermatology, pharmacy, medical entomology, and psychiatry. This demonstrates that the impact of bed bugs is of interest to a broad range of health and mental health professions and points to the potential for interdisciplinary collaboration. This is a promising approach because interdisciplinary knowledge so often leads to innovative and novel insights when examining a problem that is not yet well understood (Blackwell et al. 2009). Health professionals should be alert to the multi-faceted impacts of bed bug infestations, and take a collaborative approach with other experts, as they work thoughtfully and persistently to develop strategies that serve their clients' needs.

Our study methodology has limitations worth noting. Because this scoping review focused exclusively on peerreviewed publications, many relevant technical reports, white papers, and other gray literature with relevant data may have been excluded. Unlike systematic reviews, scoping studies by definition do not intend to assess the quality of existing literature. As well, publications included in this scoping review were limited to English-language publications. In addition, our review focused exclusively on mental health effects and, as a result, we did not examine other types of psychosocial hardships that may result from bed bug infestations, such as financial or housing insecurity.

\section{Conclusion}

Bed bugs are a public health concern of increasing scope. This scoping review demonstrates that despite considerable anecdotal knowledge, empirical evidence that clarifies the physical and mental health implications related to bed bugs is recent and limited (Goddard and de Shazo 2009). Furthermore, an important gap exists in the literature; our review demonstrates some of the types of mental health concerns associated with bed bugs; the true mental health population burden of bed bug infestations remains unknown. Although it appears likely that bed bugs present more significant mental health implications for people residing in environments more prone to bed bug infestations, rigorous empirical research is required to examine this correlation and its impacts. Further research might explore the extent to which the severity of an infestation impacts mental health, seek to clarify whether individuals who experience stronger dermatological reactions to bed bugs are at greater risk of experiencing adverse mental health effects, and examine the physical and mental health impact of the pesticides used to combat bed bugs.

The results of this scoping review reveal a clear need for research that directly addresses population concerns about bed bugs and their impacts on mental health. The lack of response to this need by researchers is concerning. However, we acknowledge that pursuing research focused on 
the mental health implications of bed bug infestations is a challenging endeavor. It is difficult to perform epidemiological studies on this topic. This is largely because exposed populations often experience stigma and marginalization, and consequently may be difficult to reach. Although the topic of bed bugs and mental health has the potential to be of interest to researchers from multiple fields, no single field currently appears to be taking a leadership role in this area. Perhaps a greater recognition of the profound mental health effects of bed bug infestations will help bolster the interest of health researchers and clinicians alike.

Acknowledgement Preparation of this article was supported by a fellowship granted to Rachelle Ashcroft, Yukari Seko, Jessica Dere, and Jaemin Kim in the Social Aetiology of Mental Illness (SAMI) training program, a Strategic Training Initiative in Health Research funded by the Canadian Institutes of Health Research (CIHR), based at the Centre for Addiction and Mental Health and the University of Toronto of which Lai Fong Chan was an affiliate fellow.

\section{References}

Anderson AL, Leffler K (2008) Bedbug infestations in the news: a picture of an emerging public health problem in the United States. J Environ Health 7:3

Arksey H, O'Malley L (2005) Scoping studies: towards a methodological framework. Int J Soc Res Methodol 8:19-32

Armed Forces Pest Management Board (2010) Bed bugs-importance, biology, and control strategies. Office of the Deputy Under Secretary of Defense for Installations \& Environment, Silver Spring

Aultman J (2012) Don't let the bedbugs bite: the Cimicidae debacle and the denial of healthcare and social justice. Med Health Care Philos 16:417-427

Baum F (2003) The new public health. Oxford University Press, Oxford

Benac N (2010) Bedbug bites becoming bigger battle. CMAJ 182:1606

Bernardeschi C, Le Cleach L, Delaunay P, Chosidow O (2013) Bed bug infestation. BMJ Open 346:1-8. doi:10.1136/bmj.f138

Blackwell A, Wilson L, Street A, Boulton C, Knell C (2009) Radical innovation: crossing knowledge boundaries with interdisciplinary teams. University of Cambridge, Cambridge

Brouqui P (2011) Arthropod-borne diseases associated with political and social disorder. Annu Rev Entomol 56:357-374

Burrows S, Perron S, Susser S (2013) Suicide following an infestation of bedbugs. Am J Case Rep 14:176-178

Calianno C (2012) Bedbugs (Cimex lectularius): identifying and managing an infestation. Nurse Pract 37:4

Canada Health (2002) Best practices, concurrent mental health and substance use disorders. Health Canada, Ottawa

Centre for Disease Control and Prevention (2011) Acute illnesses associated with insecticides used to control bed bugs-seven states, 2003-2010. MMWR Morb Mortal Wkly Rep 60:1269-1274

Chunge RN, Scott FE, Underwood JE, Zavarella KJ (1991) A pilot study to investigate transmission of headlice. Can J Public Health 82:207-208

Cimolai N, Cimolai T (2012) Otitis from the common bedbug. J Clin Aesthet Dermatol 5:43-45
Comack E, Lyons J (2011) What happens when the bed bugs do bite? The social impacts of a bed bug infestation on Winnipeg's innercity residents. Canadian Centre for Policy Alternatives, Winnipeg

Davies TG, Field LM, Williamson MS (2012) The re-emergence of the bed bug as a nuisance pest: implications of resistance to the pyrethroid insecticides. Med Vet Entomol 26:241-254

Davis S (2013) Community mental health in Canada. UBC Press, Vancouver

Delaunay P (2012) Human travel and traveling bedbugs. J Travel Med 19:6

Doggett S, Russell R (2009) Bed bugs. What the GP needs to know. Aust Fam Physician 38:880-884

Doggett S, Dwyer D, Peñas PF, Russell R (2012) Bed bugs: clinical relevance and control options. Clin Microbiol Rev 25:164-192

Durand R, Cannet A, Berdjane Z, Bruel C, Haouchine D, Delaunay P, Izra A (2012) Infestation by pyrethoids resistant bed bugs in the suburb of Paris, France. Parasite 19:381-387

Eddy C, Jones S (2011) Bed bugs, public health, and social justice: part 1, a call to action. J Environ Health 73:8-14

Fallen RS, Gooderham M (2011) Bedbugs: an update on recognition and management. Skin Ther Lett 16:2

Frydenberg A, Starr M (2003) Head lice. Aust Fam Physician 32:607-611

Gbakima AA, Terry BC, Kanja F, Kortequee S, Dukuley I, Sahr F (2002) High prevalence of bedbugs Cimex hemipterus and Cimex lectularis in camps for internally displaced persons in Freetown, Sierra Leone: a pilot humanitarian investigation. West Afr J Med 21:268-271

Gilbert SR (2011) Don't let them bite: defining the responsibilities of landlords and tenants in the event of a bedbug infestation. George Wash Law Rev 80:243-273

Goddard J, de Shazo R (2009) Bed bugs (Cimex lectularius) and clinical consequences of their bites. JAMA 301:1358-1366

Goddard J, de Shazo R (2012) Psychological effects of bed bug attacks (Cimex lectularius L.). Am J Med 125:101-103

Goforth AN, Pham AV, Carlson JS (2011) Diathesis-stress Model. In: Encyclopedia of Child Behavior and Development. Springer, US, pp 502-503

Goodall CE, Reed P (2013) Threat and efficacy uncertainty in news coverage about bed bugs as unique predictors of information seeking and avoidance: an extension of the EPPM. Health Commun 28:63-71

Grimshaw J (2010) A knowledge synthesis chapter. Canadian Institutes of Health Research, Ottawa. http://www.cihr-irsc.gc. ca/e/documents/knowledge_synthesis_chapter_e.pdf. Accessed 13 July 2015

Harlan HJ (2007) Bed bug control: challenging and still evolving. Outlooks Pest Manag Apr 18:57-61

Henner KA (2011) What are the ethical ramifications and suggested guidelines when a dental office is confirmed as having an active bedbug infestation? J Am Dent Assoc 142:1398-1399

Henriksen M (2012) Bed bug basics. Provider, October. Available at: http://www.providermagazine.com/archives/archives-2012/Pages/ 1012/Bed-Bug-Basics.aspx. Accessed 20 Mar 2013

Heukelbach J, Hengge UR (2009) Bed bugs, leeches and hookworm larvae in the skin. Clin Dermatol 27:285-290

Huntington M (2012) When bed bugs bite. J Fam Pract 61:385-388

Hurst S, Humphreys M (2011) Bedbugs: not back by popular demand. Dimens Crit Care Nurs 30:94-96

Krause-Parello CA, Sciscione P (2009) Bedbugs: an equal opportunist and cosmopolitan creature. J Sch Nurs 25:126-132

Laliberté M, Hunt M, Williams-Jones B, Feldman DE (2013) Health care professionals and bedbugs: an ethical analysis of a resurgent scourge. HEC Forum 25:245-255 
Laukhuf G (2007) Bedbugs: an old menace bites back. Regist Nurse 70:29-32

Lefferts A, Parkhill A, Cadigan D, Clayton M, Dugan-Merkler M (2009) Home Healthc Nurse 27:598-606

Levac D, Colquhoun H, O'Brien KK (2010) Scoping studies: advancing the methodology. Implement Sci 5:69

Masuda J, Zupancic T, Poland B, Cole D (2008) Environmental health and vulnerable populations in Canada: mapping an integrated equity-focused research agenda. Can Geogr 52:427-450

McKenzie MC, DeSimone IE (2012) The rise in bed bugs: prevention, management, and treatment. US Pharm 37:47-50

Mumcuoglu KY (2008) A case of imported bedbug (Cimex lectularius) infestation in Israel. Israel Med Assoc J 10:388-389

Munoz-Price L, Safdar N, Beier J, Doggett S (2012) Bed bugs in healthcare settings. Infect Control Hosp Epidemiol 33:1-7

Naumann D, Baird-Clarke CD, Ross DA (2011) Fleas on operations in Afghanistan-environmental health measures on the front line. J R Army Med Corps 157:226-228

Potter MF, Haynes KF, Connelly K, Deutsch M, Hardebeck E, Partin D, Harrison R (2010) The sensitivity spectrum: human reactions to bed bug bites. Pest Control Technol 38:70-74

Pritchard MJ, Hwang S (2009) Severe anemia from bedbugs. CMAJ 181:287-288

Prüss-Üstün A, Corvalán C (2006) Towards an estimate of the environmental burden of disease. World Health Organ, Geneva

Ralph N, Jones H, Thorpe L (2013) Self-reported bed bug infestation among New York City residents: prevalence and risk factors. J Environ Health 76:38-45

Ratnapradipa D, Ritzel D, Haramis L, Bliss K (2011) Bed bug epidemic: a challenge to public health. Am J Health Educ 42:245-251

Rieder E, Hamalian G, Maloy K, Streicker E, Sjulson L, Ying P (2012) Psychiatric consequences of actual versus feared and perceived bed bug infestations: a case series examining a current epidemic. Psychosomatics 53:85-91

Rossi L, Jennings S (2010) Bed bugs: a public health problem in need of a collaborative solution. J Environ Health 72:34-35

Sciscione P (2012) Bed bugs: they are back! The role of the school nurse in bed bug management. NASN Sch Nurse Sept 27:269-273
Shankardass K, Solar O, Freiler A, Bobbili S, Bayoumi A, O’Campo P (2011) Health in all policies: a snapshot for Ontario. Results of a realist-informed scoping review of the literature. St. Michael's Hospital, Toronto

Shum M, Comack E, Stuart E, Stuart T, Ayre R, Perron S, Beaudet S, Kosatsky T (2012) Bed bugs and public health: new approaches for an old scourge. Public Health Pract 103:399-403

Singh S, Mann BK (2013) Insect bite reactions. Indian J Dermatol Venereol Leprol 79:151-164

Stoyanova M, Hope DA (2012) Gender, gender roles, and anxiety: perceived confirmability of self report, behavioral avoidance, and physiological reactivity. J Anxiety Disord 26:206-214

Studdiford J, Conniff K, Trayes K, Tully A (2012) Bedbug infestation. Am Fam Physician 86:653-658

Susser SR, Perron S, Fournier M, Jacques L, Denis G, Tessier F, Roberge P (2012) Mental health effects from urban bed bug infestation (Cimex lectularius L.): a cross-sectional study. BMJ Open 2:e000838

Sutton D, Thomas DJ (2008) Don't let the bedbugs bite! Identifying and managing infestations. Nurse Pract 33:28-33

Ter Poorten MC, Prose NS (2005) The return of the common bedbug. Pediatr Dermatol 22:183-187

Thomas S, Wrobel M, Brown J (2013) Bedbugs: a primer for the health-system pharmacist. Am J Health Syst Pharm 70:126-130

Tompkins O (2007) Strange bedfellows. AAOHN J 55:296

Villegas SC (2012) Spinosad for the treatment of head lice infestations. Drugs Today 48:595-599

Wick JY (2011) Bedbugs, other infestations, and outbreaks: no magic bullet. Consult Pharm 26:430-434

Williams K, Willis MS (2012) Bedbugs in the 21st century: the reemergence of an old foe. Lab Med 43:141-148

Wilson KM (2011) They only come out at night: bed bugs and their alarming resurgence. Nurs Jan 41:54-58

Woodgreen Community Services (2009) Bed bugs are back. Are we ready?. Woodgreen Community Services, Toronto

Zipple AM, Batscha CL, Flaherty P, Reynolds JL (2012) Don't get bugged: practical strategies for managing bedbug infestation in psychiatric rehabilitation programs. J Psychosoc Nurs Ment Health Serv 50:22-26 